\title{
ESTRATEGIAS METODOLÓGICAS PARA EL MEJORAMIENTO DE LOS PROCESOS DE COMUNICACIÓN
}

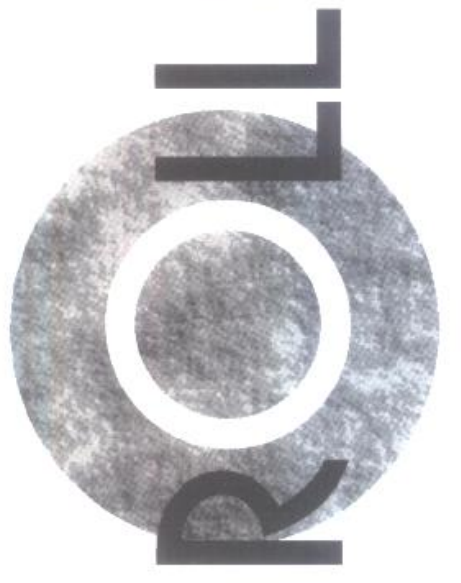

Por la directora Blanca Gladys López y los docentes: Clara Emilse Sánchez, Jaime Cruz, José Manuel Fula, Rosalba Vásquez, Rosalbina Pinto y otros*. Escuela Distrital La Gaitana (Jornada Tarde) Localidad 11 - Suba Santafé de Bogotá, D.C.
¿Por dónde empezar el cambio?

El conejo blanco se puso las gafas y preguntó: ¿por dónde debo comenzar su majestad?. Comienza desde el principio -decidió el rey- $y$ sigue hasta que llegues al final. Entonces detente.

Lewis Carroll

Cada vez que se habla de educación se llega a la conclusión que ésta debe cambiar, pero siempre esperamos que ese cambio sea externo, lo esperamos de segundos y terceros, pero nosotros ¿qué papel jugamos para que éste se dé?

"Muchos docentes se quejan del sistema educativo, de dificultades con el trabajo, de problemas en las relaciones con los alumnos, padres o los propios compañeros, pero van dejando "pasar" los años con su carga a cuestas, como si llevaran un enorme peso a las espaldas, "cada uno lleva su cruz", repetía frecuentemente así su nula aportación al cambio".

Andrés Senlle
En la Escuela Distrital de La Gaitana J.T. la reflezxión acerca del cambio educativo, evidenció que éste debía comenzar por cada uno de nosotros (los involucrados en el proceso educativo), para cambiar la actitud pasiva frente a las clases monótonas y el estar sujetos a libros que de una u otra manera nos imponían un currículo que no consultaba las necesidades de la comunidad educativa.

Fue entonces como en 1993 surgió en la institución la necesidad de crear mecanismos que mejoraran las condiciones existentes en el proceso de aprendizaje, pues la experiencia de los años anteriores, mostraba una serie de problemáticas conceptuales y metodológicas recogidas mediante estrategias de observación continua de los estudiantes. Se decide implementar el proyecto "Estrategias Metodológicas para el Mejoramiento de los Procesos de Comunicación" a través del cual se abordan los problemas existentes de forma conjunta, para poder plantear soluciones y acciones inmediatas.

Como se muestra en el Cuadro No. 1, las principales problemáticas y necesidades giraban alrededor de dos aspectos: conceptuales y procedimentales; al resolver cada uno de ellos, se orientó el conjunto de las formas de acción. 


\section{Problemáticas y Necesidades}

\section{Conceptuales Procedimentales}

Alto porcentaje de mortalidad académica.

Bajo rendimiento académico.

Se requiere preparar al estudiante para el grado sexto en:

a. Comunicación: leer, escribir, interpretar y comprender.

b. Matemáticas: suma, resta, multiplicación y división.

Los libros no cumplen con las expectativas conceptuales y metodológicas.
El niño necesita adquirir mayor seguridad en sí mismo.

Se requiere del estudiante una actitud positiva hacia la escuela y hacia el conocimiento.

Los cursos de estudiantes son muy heterogéneos, con niños avanzados, lentos, repitentes y reiniciantes.

Es indispensable crear espacios para compartir con los compañeros donde los estudiantes no se sientan superiores, ni inferiores, donde cada uno conviva sin sentirse ignorado.

\section{Formas de Acción}

Resultados Globales
- Rotación en las clases de matemáticas y español - Organización de niveles alto, medio y bajo, de acuerdo al ritmo individual de aprendizaje (lento, normal y rápido). - Integración de las diferentes áreas del conocimiento a los ejes del proyecto: habilidades comunicativas y matemáticas.

- Evaluación inicial y ubicación de los niños en los niveles.

- Observación del comportamiento social, afectivo y académico de los niños

- Elaboración de estrategias de aula de acuerdo al grupo - Determinación del mínimo de logros que se trabajan con el niño para que pueda desempeñarse en el nivel siguiente.

\author{
- Se trabaja con grupos de \\ características y \\ necesidades más \\ homogéneas. \\ - Mayor atención a los \\ problemas de los niños. \\ - Producción de material \\ didáctico y de procesos de \\ escritura en los profesores. \\ - Intercambio de ideas de \\ los alumnos en un \\ ambiente de convivencia. \\ Respeto a la opinión del \\ otro. \\ - Los estudiantes se \\ comunican con los \\ compañeros y maestros en \\ forma oral y escrita. \\ - Algunos niños quedan \\ mal ubicados desde el \\ punto de vista de su ritmo \\ académico, pero se les \\ permiten espacios que \\ resultan en buenos niveles \\ de maduración socio- \\ afectiva. \\ La comunidad de padres \\ no ha comprendido \\ completamente el PEI de \\ la Escuela La Gaitana.
}

Entre las acciones propuestas para la solución a la problemática conceptual, seguimos un proceso de consolidación de los niveles de aprendizaje; se inicia con los cursos tercero y cuarto, para ello se aplica una prueba de entrada, se ubican los niños en el nivel más favorable, se hace una observación continua de los grupos y se valora la ubicación de los nin̄os, esta etapa de la experiencia se representa en el Cuadro No. 2

Las pruebas de entrada efectuadas al iniciar el año escolar y los resultados obtenidos y con la historia que el profesor conocía del estudiante, permitió organizar tres niveles de aprendizaje en matemáticas y en lenguaje. Luego de dos meses de prueba, febrero y marzo, se observó a los estudiantes y se estudió si la organización inicial era la más conveniente.

Junto a la organización propuesta se han dado discusiones sobre la metodología empleada para la enseñanza del lenguaje y de las matemáticas, que ha permitido recoger algunos aspectos como: 


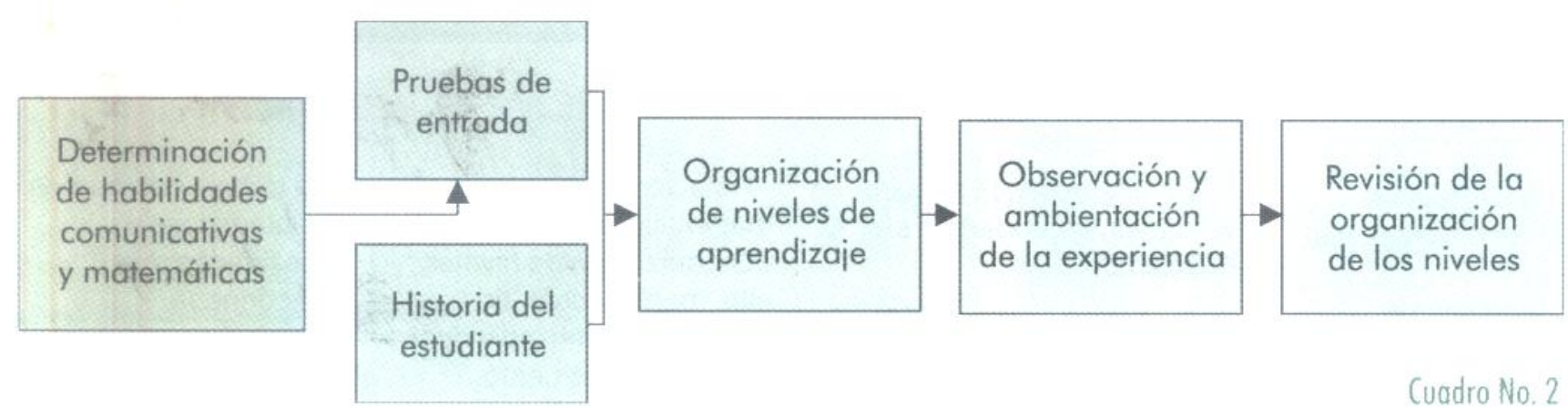

\section{Del texto tradicional al texto elaborado por maestros y alumnos}

Usualmente, el texto escolar es muy útil para maestros y alumnos porque evita "pensar": todo ya está elaborado de acuerdo a las últimas normas del Ministerio de Educación Nacional, trae parcelaciones, estan cuidadosamente seleccionados los temas, gráficos, lecturas, actividades, etc.; el maestro sólo tiene que dar la orden: "lean la página 20 y desarrollen las preguntas", "copien la página 25", "hagan los ejercicios de la página 38", "coloreen el dibujo"; muchos niños obtienen libros usados, con las respuestas a medio borrar, asi el ejercicio es mucho más fácil y el alumno que no tenga texto podrá copiarse del compañero.

Con cllo el problema que se presenta para el maestro que quiere elaborar sus propios temas, actividades y lecturas, buscando construir un saber que trascienda la escuela, no podrá hacerlo a través del texto tradicional, porque éste no cumple con las expectativas conceptuales y metodológicas del docente; él requiere de un texto más acorde con el Proyecto Educativo de la Institución, orientado hacia la solución de problemas específicos como los vividos por los estudiantes de la Escuela La Gaitana.

\section{La matemática en el aula y fuera de ella}

La "aversión" que los estudiantes sienten hacia la matemática, fruto de las dinámicas de enseñanza que en la escuela tradicional se ha impuesto, nos permite cuestionar ¿qué podriamos hacer para que no sean tan frecuentes los fracasos escolares?.
Atendiendo a la pregunta, los maestros debemos tener en cuenta la actitud del estudiante frente a las matemáticas, cambiar nuestra actitud, a veces de. arrogancia, frente a conceptos que para el maestro son sencillos y de fácil comprensión, pero para los estudiantes son difíciles y tediosos. Es importante hacer que las matemáticas sean agradables, que estén conectadas con los intereses y la vida cotidiana de los estudiantes y principalmente entendidas como otras formas de comunicación humana.

Producto de las discusiones adelantadas por los maestros', se sugirieron algunos elementos que pueden ser útiles en la enseñanza de las matemáticas:

- Exposiciones del profesor

- Discusión entre profesores y estudiantes, y entre los estudiantes

- Trabajos prácticos apropiados

- Práctica y consolidación de habilidades, destrezas y rutinas fundamentales

- Resolución de problemas, incluyendo la aplicación de la matemática a situaciones de la vida diaria.

- Trabajo investigativo.

\section{Sistematización del proceso}

Ante la necesidad de comprender la complejidad de la problemática y el proceso señalado hasta aquí, se hace necesario pensar en las rutas posibles para sistematizar el Proyecto Educativo de la Institución, al plantearnos la pregunta ¿cuáles son los puntos que hemos privilegiado en nuestras acciones educativas?; surgen así varias categorías de sistematización: alumnos, contenidos y entorno educativo, que se articulan en el manejo del lenguaje, en las relaciones interpersonales y en todas 
aquellas acciones que requieran de conocimientos matemáticos y de la comunicación en general, que contribuyen a la formación integral del individuo.

(Cuadro No. 3)

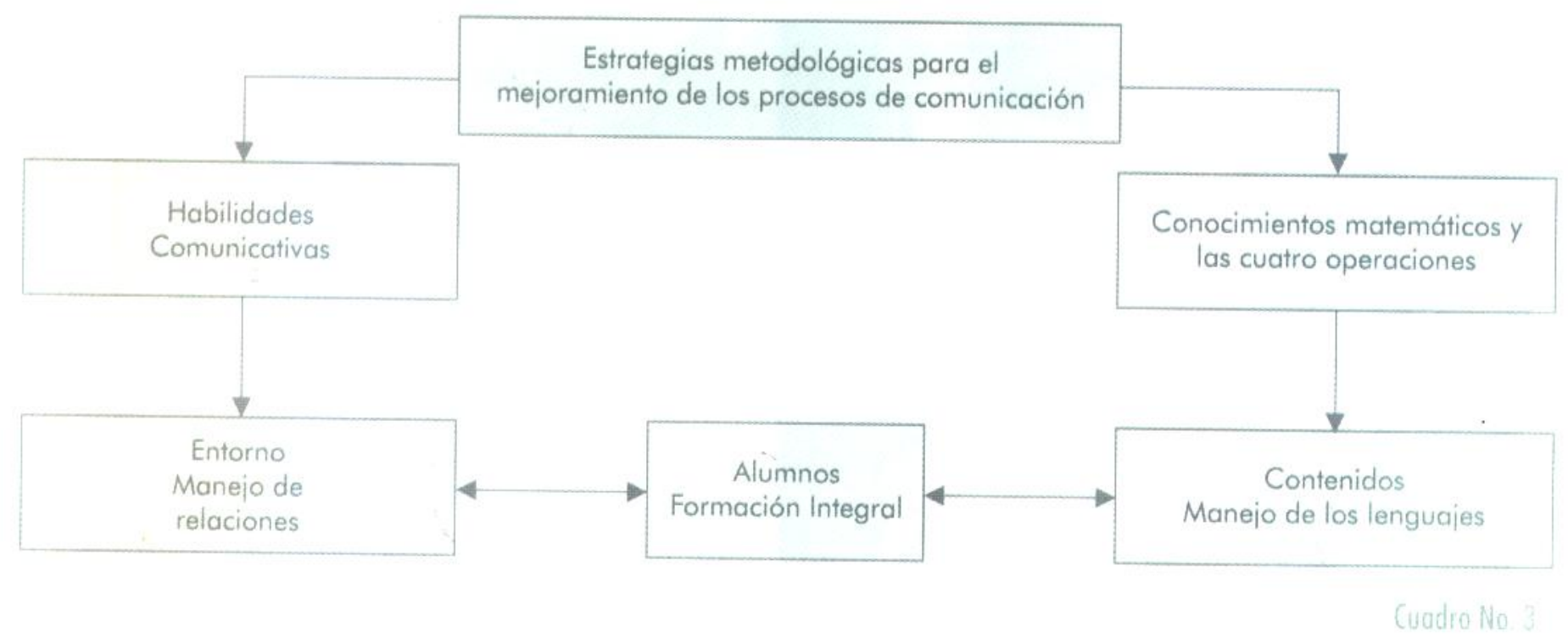

\section{Conclusiones}

Para que este proyecto se realizara fue esencial que los docentes se involucráran y se comprometieran cada vez más con su labor, ya que se requería de mayor investigación y observación de los alumnos. Hoy por hoy, esta actitud de cambio se ha visto reflejada en los maestros, es así como se percibe una mentalidad abierta y progresista.

Se han dado pautas más certeras en la ubicación de los niños por niveles. Vale anotar que a la fecha todos los cursos están articulados al proyecto y reconocemos el logro de beneficios como:

- Desarrollo de procesos de comunicación significativos para elaborar textos con contenidos de temas cotidianos.

- Los nin̄os son más conscientes de su responsabilidad en la comunidad, mostrando una actitud cordial, solidaria y ecuánime en la escuela.

- Los niños han ganado seguridad en sí mismos, autoestima y deseo de superación.

Este cambio contribuye al desarrollo educativo en nuestro país, pues el proyecto permite ver desde otra perspectiva al niño colombiano y en especial, al niño de la Escuela La Gaitana J.T. como un ser singular.

\begin{abstract}
* Concepción Gómez, Elizabeth Laguna, Gerardo Zambrano, Héctor Bejarano, Judith Riaño, Lalo Quevedo, Luz Marina Casallas, Manuel López, María Victoria Maldonado, Marina Martínez, Marleny Espinosa, Martha Janeth Aguazaco, Nelsa Vera, Sandra Jiménez, Trinidad Higuera y María Pastora Pinilla.
\end{abstract}

${ }^{1}$ Con asesoría del Anillo de Matemáticas. 